\title{
DOSE DE CAL HIDRATADA E CARACTERÍSTICAS QUÍMICAS DE UM LODO DE ESGOTO DOMÉSTICO SUBMETIDO À CALEAÇÃO
}

\author{
Mateus Pimentel de Matos ${ }^{1}$, Antonio Teixeira de Matos $^{2}$
}

\section{RESUMO}

O lodo de esgoto doméstico, após ter sido higienizado com a adição de cal, pode ter aproveitamento agrícola efetuado como corretivo de acidez e de fertilidade. Entretanto, pouco se conhece das características do lodo de esgoto após ter sido submetido à caleação. Por esta razão, objetivou-se, com a realização deste trabalho, definir as doses de cal para higienização de um lodo gerado no tratamento primário de esgoto doméstico, apresentar suas características químicas, após executada a caleação, além de obter as doses para aplicação em áreas de parques e jardins. O lodo analisado foi coletado no leito de secagem da ETE José Cirilo, Muriaé, MG, e após ser peneirado (malha $2 \mathrm{~mm}$ ), foi obtida a sua curva de incubação, adicionando-se diferentes doses $\left(0,25,50,100,150,200\right.$ e $\left.250 \mathrm{~kg} \mathrm{~m}^{-3}\right)$ de cal hidratada, $\mathrm{Ca}(\mathrm{OH})$. Para se manter o pH ligeiramente superior a 12, por mais de $2 \mathrm{~h}$ após ter sido realizada a mistura, a dose de cal a ser aplicada deve ser de 43,2 $\mathrm{kg} \mathrm{m}^{-3}$, e por $72 \mathrm{~h}$ deve ser de 71,2 $\mathrm{kg} \mathrm{m}^{-3}$. Aplicada a dose de 71,2 $\mathrm{kg} \mathrm{m}^{-3}$ no lodo, o material foi submetido à digestão nítrico-perclórico para quantificação das concentrações totais de $\mathrm{N}, \mathrm{P}, \mathrm{K}, \mathrm{Ca}, \mathrm{Fe}, \mathrm{Na}, \mathrm{Mg}$, $\mathrm{Cr}, \mathrm{Ni}, \mathrm{Cd}, \mathrm{Pb}, \mathrm{Cu}, \mathrm{Mn}$ e Zn. Os valores obtidos, em $\mathrm{g} \mathrm{kg}^{-1}$, foram de 10,08 de N; 3,63 de P; 2,80 de K; 3,16 de Ca; e 52,53 de Fe, enquanto que, em mg kg-1, foram de 268,75 de $\mathrm{Mg}$; 33,50 de Ni; 2,25 de Pb; 119,75 de Cu; 499,00 de Mn; e 395,50 de Zn. O Cd e o Cr não foram detectados.

Palavras-chave: higienização, resíduos sólidos, tratamento sanitário

\section{ABSTRACT \\ DOSE OF LIME AND CHEMICAL CHARACTERISTICS OF LIMED SEWAGE SLUDGE}

The sewage sludge, after being sanitized with the addition of lime, can be used to correct soil acidity and improve fertility. However, little is known about the post-liming characteristics of sewage sludge. This work was done to define the dose of lime for hygienization of sludge generated during the primary treatment of domestic sewage, determine chemical characteristics after liming, and determine application rate for use in parks and gardens. The sludge was collected from sewage drying bed of treatment Plant José Cirilo, Muriaé, MG. After sieving (2 mm mesh) an incubation curve was generated by adding different doses $\left(0,25,50,100,150,200\right.$ and $\left.250 \mathrm{~kg} \mathrm{~m}^{-3}\right)$ of hydrated lime. The data revealed that to keep the $\mathrm{pH}$ slightly above 12 for more than $2 \mathrm{~h}$, liming dose should be $43.20 \mathrm{~kg} \mathrm{~m}^{3}$ and if this $\mathrm{pH}$ is to be maintained for $72 \mathrm{~h}$ the application rate should be increased to $71.20 \mathrm{~kg} \mathrm{~m}^{-3}$. The sludge treated at the rate of $71.2 \mathrm{~kg} \mathrm{~m}^{-3}$, was subjected to nitric-perchloric digestion to quantify total $\mathrm{N}, \mathrm{P}, \mathrm{K}, \mathrm{Ca}, \mathrm{Fe}, \mathrm{Na}, \mathrm{Mg}, \mathrm{Cr}$, $\mathrm{Ni}, \mathrm{Cd}, \mathrm{Pb}, \mathrm{Cu}, \mathrm{Mn}$ and $\mathrm{Zn}$. The values, in $\mathrm{g} \mathrm{kg}^{-1}$, were 10.08 of $\mathrm{N}, 3.63$ of $\mathrm{P}, 2.80$ of $\mathrm{K}, 3.16$ of $\mathrm{Ca}$ and 52.53 of Fe, while, in $\mathrm{mg} \mathrm{kg}^{-1}, 268.75$ of $\mathrm{Mg}, 33.50$ of $\mathrm{Ni}, 2.25 \mathrm{of} \mathrm{Pb}, 119.75$ of $\mathrm{Cu}, 499.00$ of $\mathrm{Mn}$ and 395.50 of $\mathrm{Zn}$. The $\mathrm{Cd}$ and Cr were not detected.

Keywords: sanitation, solid waste, sanitary treatment

\section{Recebido para publicação em 21/11/2011. Aprovado em 24/05/2012.}

1- Engenheiro Agrícola e Ambiental, Doutorando em Saneamento, UFMG, Belo Horizonte-MG:, mateus.matos@ufv.br 2- Engenheiro Agrícola, Prof. Associado, Depto. de Engenharia Agrícola, UFV, Viçosa - MG 


\section{INTRODUÇÃO}

O aproveitamento agrícola do lodo de esgoto apresenta-se com uma das alternativas mais viáveis para disposição final deste resíduo, em vista dos benefícios que ele pode trazer ao solo. A aplicação desses biossólidos aumenta a absorção de nutrientes pelas culturas, com ganho de produtividade, desde que a adubação seja feita em doses adequadas, já que, caso isso não seja observado, há o risco de salinização e/ou, contaminação do solo e de águas subterrâneas e superficiais. Em razão de sua riqueza em termos de nutrientes e de matéria orgânica a disposição do lodo no solo é uma das alternativas técnicas e ambientalmente viáveis, considerando-se a pobreza química generalizada dos solos brasileiros, além de proporcionar maior vida útil aos aterros sanitários, que deixariam de ser ocupados com este resíduo (FIA et al., 2006).

Entretanto, ao se aplicar lodo de esgoto doméstico no solo, torna-se necessária a sua higienização prévia, a fim de se evitar riscos sanitários aos agricultores e consumidores dos produtos agrícolas ou usuários das áreas receptoras do resíduo. O risco sanitário proporcionado pelo lodo pode ser minimizado por meio da adoção de técnicas de higienização que concorram para a eliminação dos organismos patogênicos presentes (ANDREOLI, 2001). Ainda, segundo o autor, dentre os processos de higienização e estabilização do lodo de esgoto, a caleação é um dos mais difundidos, o que é decorrente, principalmente, do seu baixo custo e facilidade operacional.

Na caleação, um tratamento químico é realizado com a adição da cal ao lodo, de forma a elevar seu $\mathrm{pH}$ até valores superiores a 12 , condição na qual ocorre a inativação ou destruição de grande parte dos agentes patogênicos, além de proporcionar a estabilização química e redução do odor do lodo (SANEPAR, 1997). A dose de cal hidratada para higienização do lodo de esgoto, suficiente para, após 3 dias de incubação, manter o pH ligeiramente superior a 12, deve estar, segundo Malina (1993), citado por Pinto (2001), entre 54 e $154 \mathrm{~kg} \mathrm{t}^{-1}$. Fia et al. (2005), obtiveram para lodo proveniente de lagoa de maturação que serve como pós tratamento para o esgoto do Condomínio Bosque Acamari, Viçosa, curva de incubação com cal hidratada, para se obter pH 12, após 72 h de reação, e definiram a dose de $75 \mathrm{~kg} \mathrm{t}^{-1}$ de cal hidratada no lodo.

Após a caleação, o lodo se torna básico e, por esta razão deverá ser usado como corretivo de acidez de solo. Como a maior parte dos solos brasileiros é ácida, faz-se necessário a aplicação de corretivos para aumentar o seu potencial produtivo. Desta forma, o uso de lodo caleado pode substituir a aplicação de calcário nos solos, concorrendo para aumento de sua fertilidade.

Fia et al. (2006), ao efetuarem a caracterização química do solo, antes e após ter recebido o lodo de esgoto caleado, verificaram grande melhoria na sua fertilidade, com aumento no $\mathrm{pH}$ e na disponibilidade de nutrientes e, com isso, aumento na produtividade de milho. Os mesmos autores concluíram que nem mesmo a aplicação das maiores doses de lodo de esgoto caleado proporcionou concentrações de metais pesados, nos solos, que suplantassem os limites recomendados pela legislação ambiental, sendo o crômio o único, entre os metais pesados avaliados, que apresentou concentração crescente com o aumento da dose aplicada.

Com a realização deste trabalho, objetivouse obter a dose de cal hidratada a ser utilizada na higienização do lodo de esgoto doméstico produzido na ETE José Cirilo, além das suas características químicas, após caleado, seu valor como corretivo e fertilizante para o solo, bem como do risco ambiental que pode proporcionar para áreas de parques e jardins do município de Muriaé-MG.

\section{MATERIAL E MÉTODOS}

O lodo foi coletado no leito de secagem da Estação de Tratamento de Esgotos José Cirilo, localizada em Muriaé, MG, sendo que o material, na ocasião da coleta, encontrava-se em secagem a 75 dias.

O presente estudo pode ser divido em etapas, sendo elas: higienização do lodo, definição das doses de lodo caleado para correção do $\mathrm{pH}$ dos solos em estudo e análise química do lodo caleado, com avaliação de potencial de contaminação do solo.

A curva de incubação do lodo com cal hidratada foi realizada no Laboratório de Solos e Resíduos 
Sólidos do Departamento de Engenharia Agrícola (DEA), Universidade Federal de Viçosa (UFV), sendo obtida com a aplicação de diferentes doses de cal hidratada, $\mathrm{Ca}(\mathrm{OH})_{2},(0,25,50,100,150,200$ e $250 \mathrm{~kg} \mathrm{~m}^{-3}$ ) no material secado ao ar e peneirado (peneira $2 \mathrm{~mm}$ ).

A mistura recebeu água até ser atingida a capacidade de campo, para favorecimento da reação e, após 2 h e 72 h de efetuada a mistura, o $\mathrm{pH}$ do material foi medido em suspensão preparada com solução $\mathrm{CaCl}_{2}$ 0,01 mol L-1 (MATOS, 2008).

Uma curva do $\mathrm{pH}$ em função da dose de cal foi obtida, para cada tempo após efetuada a mistura. Assim, foram obtidas as menores doses para obtenção de um pH igual a 12. Equações matemáticas foram ajustadas, por regressão, aos dados obtidos no intervalo no qual ocorreu a maior variação do $\mathrm{pH}$ com a dose de cal, tendo em vista que a curva atinge um platô, no qual o $\mathrm{pH}$ permanece próximo de 13, independente da dose de cal aplicada. As equações de maior coeficiente de determinação foram as escolhidas para descrição matemática do fenômeno em estudo.

Para a caracterização química e definição das doses de aplicação do lodo caleado no solo, foram coletadas amostras nas seguintes praças da cidade de Muriaé, na Zona da Mata Mineira: Carlos Drummond de Andrade, localizada no Bairro Barra (Drum); Dornellas, no Bairro Dornellas (Dorn) e Franco Suíço, no Bairro Santana (Sant). Além de tais pontos, amostras de solo de dois locais do Horto também tiveram suas características avaliadas, sendo estes solos (Horto 1 e Horto 2) utilizados como substrato para a produção de mudas de espécies vegetais arbóreas, de interesse para o setor de parques e jardins do município.

Análise textural do solo foi realizada com a separação das suas frações areia, silte e areia, além do cálculo do teor de água no solo para usar como fator de correção no cálculo da proporção granulométrica. A separação das frações foi efetuada depois da dispersão física e química das amostras de solo, e posteriormente por passagem em peneira e por decantação, no método da Pipeta (EMBRAPA, 1997).

$\mathrm{Na}$ caracterização química dos solos, foram realizadas as seguintes determinações: fósforo disponível; cálcio e magnésio trocáveis e alumínio trocável; sódio e potássio trocáveis e $\mathrm{pH}$, seguindose métodos descritos pela EMBRAPA (1997). A concentração de fósforo disponível foi quantificada utilizando-se o espectrofotômetro; a de cálcio e magnésio trocáveis, por titulação com EDTA; a de alumínio trocável por titulação com $\mathrm{NaOH}$ e a de sódio e potássio, por fotometria de chama. Todas as análises foram conduzidas no Laboratório de Solos e Resíduos Sólidos do DEA/UFV.

Para se caracterizar, em termos químicos, o lodo caleado e, assim, calcular a quantidade de macro e micronutrientes e metais pesados aplicados no solo, considerando-se a dose de lodo caleado definida para cada solo, realizou-se análise da concentração total desses elementos químicos no material. $\mathrm{O}$ nitrogênio total foi quantificado utilizando-se o método Kjeldahl modificado, com a introdução do ácido salicílico para inclusão das formas nítricas (MATOS, 2012). Após digestão nítrico-perclórico das amostras, o potássio e o sódio foram quantificados utilizando-se fotômetro de chama, o fósforo por espectrofotômetro e os demais elementos químicos por espectrofotometria por absorção atômica, sendo todas as análises conduzidas conforme recomendado no Standard Methods for the Examination. of Water and Wastewater (APHA et al., 2005).

Para avaliação do potencial de contaminação do solo, comparou-se os valores que serão disponibilizados pela aplicação do lodo caleado, com a Resolução CONAMA no 375 de 2006, que regulamenta a aplicação de biossólidos de esgoto, quanto a disponibilização de metais pesados.

\section{RESULTADOS E DISCUSSÃO}

O lodo analisado apresentou massa específica igual a $454,5 \mathrm{~kg} \mathrm{~m}^{-3}$ e os resultados de medição do $\mathrm{pH}$, medidos após 2 e 72 horas, em função das diferentes doses de cal hidratada aplicadas, estão apresentados na Tabela 1. A partir dos valores de $\mathrm{pH}$ como função da dose de cal, foram obtidas as curvas e as equações apresentadas nas Figuras 1a (2 h) e $1 b$ (72 h).

A análise de tais equações e gráficos indica que, para se manter o $\mathrm{pH}$ ligeiramente superior a 12, por $2 \mathrm{~h}$ após realizada a mistura, a dose de cal a ser aplicada no lodo deve ser de $29,1 \mathrm{~kg} \mathrm{~m}^{-3}$; até 72 
$\mathrm{h}$ a dose a ser aplicada deve ser de $71,2 \mathrm{~kg} \mathrm{~m}^{-3}$ de lodo, o que corresponde a $64,10 \mathrm{~kg} \mathrm{t}^{-1}$ e 156,70 $\mathrm{kg} \mathrm{t}^{-1}$, respectivamente. Para dose de cal hidratada a ser aplicada em lodo de esgoto doméstico, a primeira dose está no intervalo, enquanto a outra está pouco acima conforme apresentado por Malina (1993), citado por Fia et al. (2005). Os resultados são confirmatórios de que a dose de cal deva aumentar com o tempo de reação, tendo em vista que deverá neutralizar a acidez gerada no processo de degradação biológica. Moreira et al. (2006), verificaram que a dose mínima para se atingir valor de $\mathrm{pH} 12$, em 72 horas de reação, foi de $19,4 \mathrm{~kg} \mathrm{~m}^{-3}$. Esse valor é menor que o obtido neste trabalho, entretanto, há de se considerar que os referidos autores avaliaram a caleação em lodo de esgoto doméstico coletado no fundo de uma lagoa de estabilização, material que esteve submetido a muito tempo de degradação bioquímica.

A caracterização dos solos torna-se necessária para a definição das doses de lodo caleado que serão aplicados. Na Tabela 2 estão apresentados os resultados da análise granulométrica dos solos avaliados.

De acordo com a porcentagem das frações, os solos $\mathrm{H}_{1}, \mathrm{H}_{2}$, Dorn e Sant podem ser classificados como muito argilosos, e Drum, como argila. As variáveis químicas analisadas nos solos antes da aplicação do lodo caleado estão apresentadas na Tabela 3.

$\mathrm{Na}$ Figura 2a, 2b e 2c estão apresentadas, respectivamente, as curvas e equações ajustadas parapHem função da dose aplicada de lodo caleado, nas amostras de solo $\mathrm{H}_{1}, \mathrm{H}_{2}$ e Drum. Amostras dos outros solos (Dorn e Sant) não foram analisadas
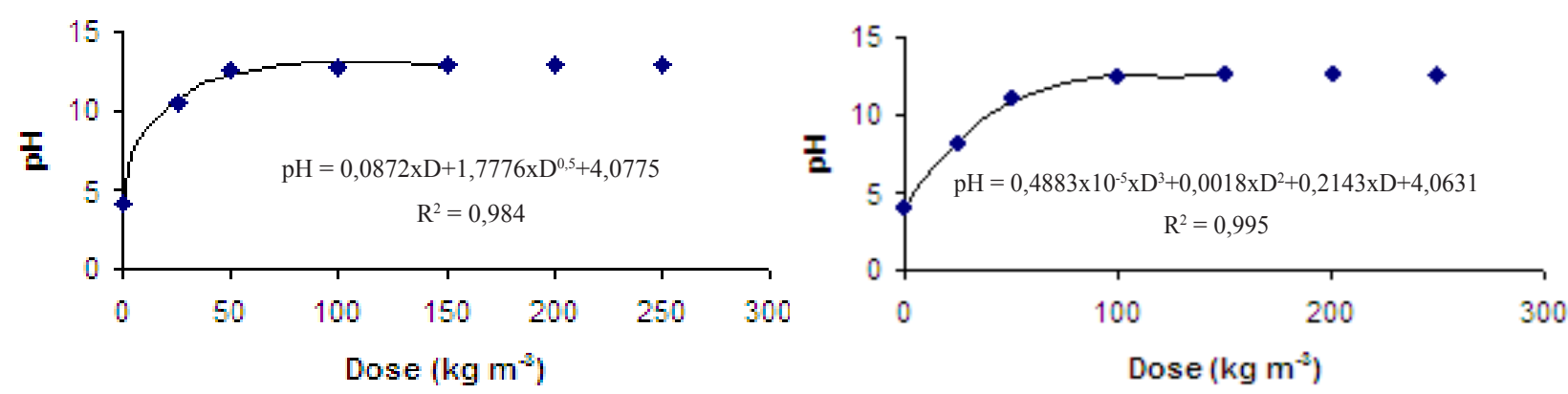

Figura 1. Curva de incubação do lodo de esgoto com cal hidratada, com medição do pH após 2 (a) e 72 horas (b) de realização da mistura.

Tabela 1. Valores de pH do lodo após 2 h e 72 h de incubação com cal hidratada

\begin{tabular}{|c|c|c|}
\hline Dose & pH (2 h) & pH (72 h) \\
\hline Natural & 4,09 & 4,12 \\
\hline $25 \mathrm{~kg} \mathrm{~m}^{-3}$ & 10,58 & 8,20 \\
\hline $50 \mathrm{~kg} \mathrm{~m}^{-3}$ & 12,68 & 11,10 \\
\hline $100 \mathrm{~kg} \mathrm{~m}^{-3}$ & 12,83 & 12,48 \\
\hline $150 \mathrm{~kg} \mathrm{~m}^{-3}$ & 12,88 & 12,56 \\
\hline $200 \mathrm{~kg} \mathrm{~m}^{-3}$ & 12,90 & 12,60 \\
\hline $250 \mathrm{~kg} \mathrm{~m}^{-3}$ & 12,90 & 12,62 \\
\hline
\end{tabular}

Tabela 2. Composição granulométrica dos solos das diferentes áreas.

\begin{tabular}{cccc}
\hline & Areia $\left(\mathbf{d a g} \mathbf{~ k g}^{-1}\right)$ & Silte $\left(\mathbf{d a g} \mathbf{~ k g}^{-1}\right)$ & Argila $\left(\mathbf{d a g} \mathbf{~ k g}^{-\mathbf{1}}\right)$ \\
\hline $\mathrm{H}_{1}$ & 23,40 & - & 76,60 \\
$\mathrm{H}_{2}$ & 33,86 & - & 66,14 \\
Drum & 35,14 & 5,96 & 58,90 \\
Dorn & 34,54 & - & 65,46 \\
Sant & 29,79 & 9,06 & 61,15 \\
\hline
\end{tabular}


por apresentarem, naturalmente, $\mathrm{pH}$ maior que 5,5. A partir das equações ajustadas, calculou-se as doses de lodo caleado que proporcionassem elevação do $\mathrm{pH}$ para até 6,5 , valor este considerado adequado para solos agrícolas. Segundo Fia et al. (2005), na faixa de $\mathrm{pH}$ de 5,5 a 6,5 proporciona-se a disponibilização de muitos macro $(\mathrm{P}, \mathrm{Ca}, \mathrm{S}, \mathrm{N}$ e $\mathrm{K}$ ) e micronutrientes ( $\mathrm{B}, \mathrm{Mo}, \mathrm{Cl}$ e outros) além de redução, no caso de problemas de excesso, da disponibilidade de $\mathrm{Cu}, \mathrm{Fe}, \mathrm{Mn}, \mathrm{Zn}$ e $\mathrm{Al}$, os quais poderiam trazer efeitos tóxicos às plantas. Assim, considerando-se a correção do $\mathrm{pH}$ até 6,5, as aplicações recomendadas para os solos $\mathrm{H}_{1}, \mathrm{H}_{2}$ e Drum seriam, respectivamente de 16,$24 ; 19,27$ e $12,64 \mathrm{tha}^{-1}$.

As características químicas do lodo caleado, considerando a dose de cal necessária para manter o pH ligeiramente superior a 12 no lodo de esgoto por $72 \mathrm{~h}$ após realizada a mistura $(71,2 \mathrm{~kg}$ de cal por $\mathrm{m}^{3}$ de lodo), estão apresentadas na Tabela 4.

Tabela 3. Caracterização química dos solos analisados.

\begin{tabular}{lccccc}
\hline Variável & $\mathbf{H}_{\mathbf{1}}$ & $\mathbf{H}_{\mathbf{2}}$ & Drum & Dorn & Sant \\
\hline $\mathrm{P}\left(\mathrm{mg} \cdot \mathrm{dm}^{-3}\right)$ & 7,79 & 6,84 & 10,64 & 11,40 & 9,88 \\
$\mathrm{~K}^{+}\left(\mathrm{cmol}_{\mathrm{c}} \cdot \mathrm{dm}^{-3}\right)$ & 0,12 & 0,06 & 0,18 & 0,22 & 0,21 \\
$\mathrm{Na}^{+}\left(\mathrm{cmol}_{\mathrm{c}} \cdot \mathrm{dm}^{-3}\right)$ & 0,04 & 0,04 & 0,04 & 0,04 & 0,13 \\
$\mathrm{Ca}^{2+}+\mathrm{Mg}^{2+}\left(\mathrm{cmol}_{\mathrm{c}} \cdot \mathrm{dm}^{-3}\right)$ & 0,60 & 0,12 & 2,24 & 1,68 & 2,16 \\
$\mathrm{Al}^{3+}\left(\mathrm{cmol}_{\mathrm{c}} \cdot \mathrm{dm}^{-3}\right)$ & 0,49 & 0,59 & 0,20 & 0,29 & 0,00 \\
$\mathrm{pH}$ & 4,61 & 4,53 & 5,38 & 6,02 & 6,80 \\
$\mathrm{SB}\left(\mathrm{cmol}_{\mathrm{c}} \cdot \mathrm{dm}^{-3}\right)$ & 0,76 & 0,22 & 2,46 & 1,94 & 2,50 \\
$\mathrm{t}\left(\mathrm{cmol}_{\mathrm{c}} \cdot \mathrm{dm}^{-3}\right)$ & 1,25 & 0,81 & 2,66 & 2,23 & 2,50 \\
$\mathrm{~m}(\%)$ & 39,20 & 72,83 & 7,52 & 13,00 & 0,00 \\
\hline
\end{tabular}

Em que: SB - Soma de bases; $\mathrm{t}$ - CTC efetiva e $\mathrm{m}$ - saturação por $\mathrm{Al}^{3+}$.
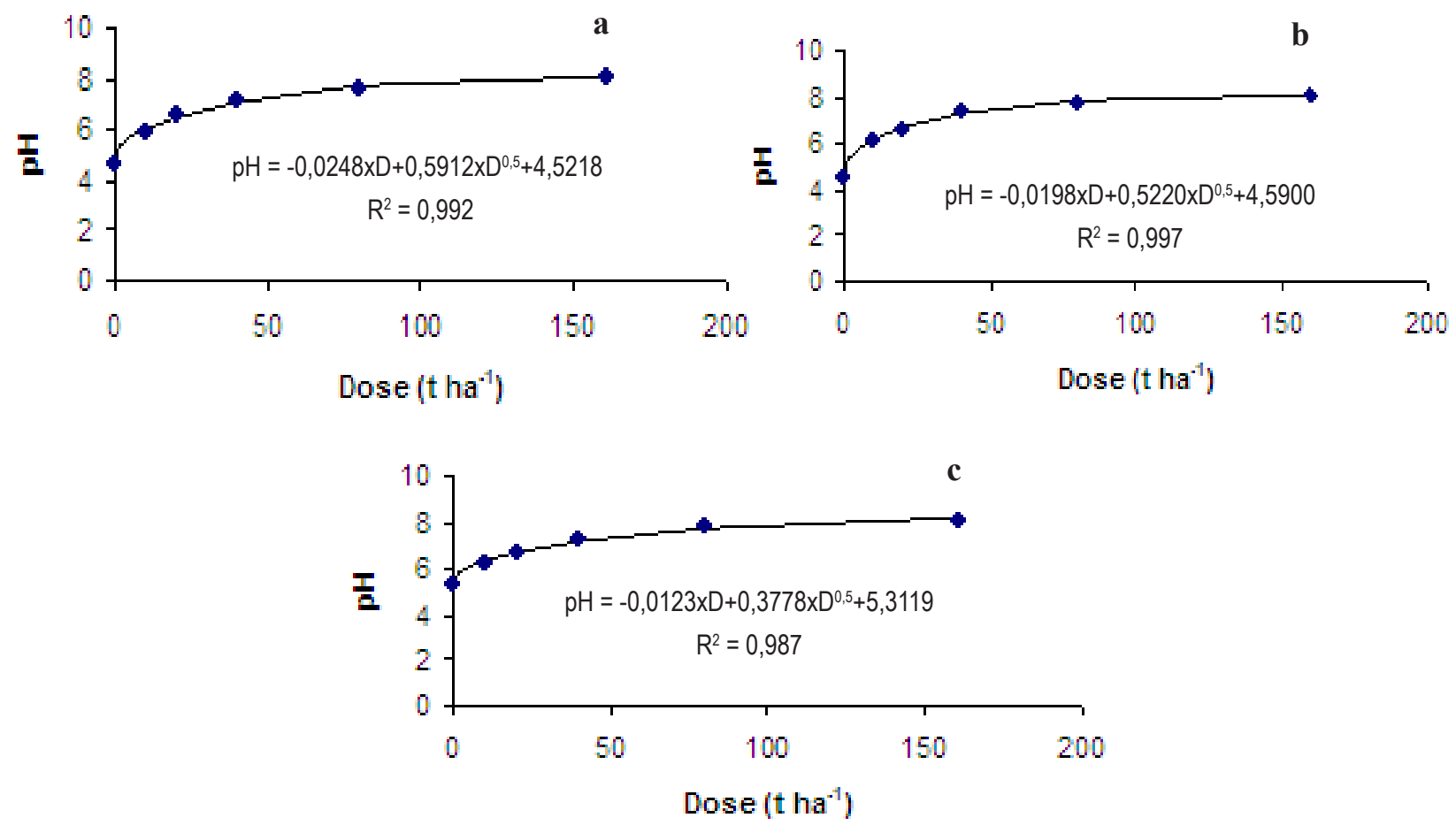

Figura 2. Curvas de incubação do solo do Horto Florestal - $\mathrm{H}_{1}$ (a), Horto Florestal - $\mathrm{H}_{2}$ (b) e da Praça Carlos Drummond de Andrade (c) com o lodo caleado 
Analisando-se os dados obtidos com padrões estabelecidos na Resolução 375/2006 do CONAMA, verifica-se que a contaminação com elementos químicos perigosos será baixa, ficando muito aquém da concentração máxima permitida para o lodo de esgoto doméstico. Com isso, a aplicação correta do lodo caleado é segura no que se refere aos metais cádmio, chumbo, cobre, crômio, níquel e zinco.

A quantidade de macro e micronutrientes aplicada ao solo, quando da aplicação das doses de 16,$24 ; 19,27$ e $12,64 \mathrm{t} \mathrm{ha}^{-1}$ de lodo caleado, respectivamente para os solos $\mathrm{H}_{1}, \mathrm{H}_{2}$ e Drum, está apresentada na Tabela 5 .

Uma vez que a camada de solo que é considerada de mistura de um adubo ou corretivo de $\mathrm{pH}$ é $0,2 \mathrm{~m}$, tem-se, assim, $81,85 \mathrm{~g} \mathrm{~m}^{-3}$ de $\mathrm{N}, 29,5 \mathrm{~g} \mathrm{~m}^{-3}$ de $\mathrm{P}$ e $22,75 \mathrm{~g} \mathrm{~m}^{-3}$ de $\mathrm{K}$ são as concentrações proporcionadas ao solo pela aplicação das referidas doses de lodo caleado, no solo $\mathrm{H}_{1}$, e $97,10 \mathrm{~g} \mathrm{~m}^{-3}$ de $\mathrm{N}, 35,0 \mathrm{~g} \mathrm{~m}^{-3}$ de $\mathrm{Pe}$ $27,0 \mathrm{~g} \mathrm{~m}^{-3}$ de $\mathrm{K}$, no solo $\mathrm{H}_{2}$. Em relação ao solo Drum, como é proveniente de uma área de praça pública, constituída por gramado e jardins, foi avaliada a aplicação por área superficial, sendo as doses de N, P e K de, respectivamente, 12,7; 4,0 e $3,5 \mathrm{~g} \mathrm{~m}^{-2}$. Não foram encontrados traços de cádmio ou crômio no lodo caleado, portanto estes elementos químicos não deverão ser motivo de preocupação quando da aplicação das doses definidas de lodo caleado no solo. Quanto aos outros metais, as doses aplicadas proporcionaram doses ínfimas, bem aquém das permitidas pela Resolução CONAMA 375/2006. Além disso, como a caleação é feita de 4 em 4 anos, as doses aplicadas ficam ainda mais diluídas, devendose levar muito tempo para que haja acúmulo suficiente desses poluentes. Assim, o risco de contaminação do solo é baixo e o tempo para que limites estabelecidos na Resolução CONAMA 375/2006 possam ser superados é muito grande. Além disso, sabe-se que no solo não há grande disponibilidade desses metais e ainda, com o tempo, ocorre sua complexação e quelação pelo material orgânico e adsorção específica na fração mineral (MATOS, 2010), soma-se, ainda, o aumento no $\mathrm{pH}$ do meio, diminuindo ainda mais os riscos de contaminação ambiental.

Tabela 4. Concentrações totais de macro e micronutrientes e metais pesados na matéria seca do lodo caleado

\begin{tabular}{|c|c|c|c|c|c|c|c|c|c|c|c|c|}
\hline $\mathbf{N}$ & $\mathbf{P}$ & $\mathbf{K}$ & $\mathrm{Ca}$ & $\mathbf{F e}$ & Mg & $\mathbf{N i}$ & $\mathbf{P b}$ & $\mathrm{Cu}$ & Mn & Zn & Cd & $\mathrm{Cr}$ \\
\hline -------. & ---- & $\mathrm{g} \mathrm{kg}^{-1}$ & ------ & ----- & -------- & ------ & 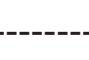 & ---- mg k & $\sigma^{-1}=---$ & -------- & ------. & ---- \\
\hline 10,08 & 3,63 & 2,80 & 3,16 & 52,53 & 268,75 & 33,50 & 2,25 & 119,75 & 499,0 & 395,5 & N.D. & N.D. \\
\hline
\end{tabular}

Tabela 5. Quantidade de macro e micronutrientes e metais pesados adicionados ao solo com a aplicação das doses de lodo caleado definidas para os solos $\mathrm{H}_{1}, \mathrm{H}_{2}$ e Drum

\begin{tabular}{cccc}
\hline Elemento químico & Solo $\mathbf{H}_{1}$ & Solo $\mathbf{H}_{2}$ & Solo Drum \\
\hline $\mathrm{N}\left(\mathrm{kg} \mathrm{ha}^{-1}\right)$ & 163,7 & 194,2 & 127,4 \\
$\mathrm{P}\left(\mathrm{kg} \mathrm{ha}^{-1}\right)$ & 59,0 & 70,0 & 45,9 \\
$\mathrm{~K}\left(\mathrm{~kg} \mathrm{ha}^{-1}\right)$ & 45,5 & 54,0 & 35,4 \\
$\mathrm{Ca}\left(\mathrm{kg} \mathrm{ha}^{-1}\right)$ & 51,3 & 60,9 & 39,9 \\
$\mathrm{Fe}\left(\mathrm{kg} \mathrm{ha}^{-1}\right)$ & 853,1 & 1012,3 & 664,0 \\
$\mathrm{Mg}\left(\mathrm{g} \mathrm{ha}^{-1}\right)$ & 4364,5 & 5178,8 & 3397,0 \\
$\mathrm{Ni}\left(\mathrm{g} \mathrm{ha}^{-1}\right)$ & 544,0 & 645,6 & 423,4 \\
$\mathrm{~Pb}\left(\mathrm{~g} \mathrm{ha}^{-1}\right)$ & 36,5 & 43,4 & 28,4 \\
$\mathrm{Cu}\left(\mathrm{g} \mathrm{ha}^{-1}\right)$ & 1944,7 & 2307,6 & 1513,6 \\
$\mathrm{Mn}\left(\mathrm{g} \mathrm{ha}^{-1}\right)$ & 8103,8 & 9615,7 & 6307,4 \\
$\mathrm{Zn}\left(\mathrm{g} \mathrm{ha}^{-1}\right)$ & 6422,9 & 7621,3 & 4999,1 \\
$\mathrm{Cd}\left(\mathrm{g} \mathrm{ha}^{-1}\right)$ & N.D. & N.D. & N.D. \\
$\mathrm{Cr}\left(\mathrm{g} \mathrm{ha}^{-1}\right)$ & N.D. & N.D. & N.D. \\
\hline
\end{tabular}




\section{CONCLUSÕES}

- Na caleação do lodo de esgoto doméstico produzido na ETE José Cirilo, Muriaé-MG, a dose de cal hidratada deve ser de $29,1 \mathrm{~kg} \mathrm{~m}^{-3}$, considerando-se um tempo de reação de $2 \mathrm{~h}$, e de $71,2 \mathrm{~kg} \mathrm{~m}^{-3}$, considerando-se um tempo de reação superior a $72 \mathrm{~h}$;

- Recomenda-se a aplicação das doses de 16,$24 ; 19,27$ e $12,64 \mathrm{t} \mathrm{ha}^{-1}$ de lodo caleado, respectivamente para os solos $\mathrm{H}_{1}, \mathrm{H}_{2}$ e Drum, com o intuito de proporcionar elevação do $\mathrm{pH}$ até 6,5 ;

- O lodo caleado atende à legislação ambiental (Resolução CONAMA375/2006), apresentando baixas concentrações de metais pesados, podendo ser utilizado, numa mesma área, por longo período de tempo, sem que venha a suplantar as cargas máximas estabelecidas na referida legislação ambiental; $\mathrm{e}$

- A aplicação única e exclusiva do lodo de esgoto caleado, estabelecida como corretivo de acidez do solo, melhora a fertilidade dos solos com vários macro e micronutrientes, embora possa não ser suficiente par atender as necessidades de $\mathrm{K}$ e $\mathrm{P}$ das culturas.

\section{REFERÊNCIAS BIBLIOGRÁFICAS}

APHA, AWWA, WEF - American Public Health Association. Standard methods for the examination of water and wastewater. $21^{\text {st. }}$ ed. Washington: APHA, AWWA, WEF, 2005. s.n.p.

ANDREOLI, C.V. (Coord.) Resíduos sólidos do saneamento: Processamento, reciclagem e disposição final. Rio de Janeiro: Rima, ABES, 2001. 282p.

BRASIL. CONAMA. Ministério do Meio Ambiente. Deliberação Normativa 375, 2006. 32p. Disponível em: <http://www.comitepcj.
sp.gov.br/download/Res-CONAMA-375-06.pdf>, Acesso em setembro de 2011.

EMBRAPA/CNPS. Manual de métodos de análise de solo. Rio de Janeiro: Centro Nacional de Pesquisa de Solos, 2. ed., 1997212 p. (EMBRAPACNPS. Documentos; 1)

FIA, R.; MATOS, A.T. de; AGUIRRE, C.I. Características Químicas de solo adubado com doses crescentes de lodo de esgoto caleado. Engenharia na Agricultura, Viçosa, MG, v.13, n.4, p.287-299, 2005.

FIA, R.; MATOS, A.T. de; AGUIRRE, C. I. Produtividade e concentração de nutrientes e metais pesados em milho adubado com doses crescentes de lodo de esgoto caleado. Engenharia na Agricultura, Viçosa, MG, v.14, n.1, p.287-299, 2006.

MATOS, A.T. Qualidade do Meio Físico Ambiental: Práticas de Laboratório. Viçosa: Ed. UFV, 2012. 150p.

MATOS, A.T. Poluição ambiental - Impactos no meio físico. Viçosa, MG: Ed. UFV, 2010. 260p.

MOREIRA D.A.; FARAGE, J. A. P., CHAGAS, R. C.; LIMA, C. R. C. MATOS, A. T. Curva de incubação do lodo primário de esgoto doméstico. Simpósio Mineiro de Engenharia Ambiental, Viçosa, 2006. Anais..., Viçosa, 2006 (CD-Rom)

PINTO, M.T. Higienização de lodos. In: ANDREOLI, C.V.; von SPERLING, M.; FERNANDES, F. Lodo de esgotos: tratamento e disposição final. Belo Horizonte: DESA/UFMG; SANEPAR. 2001. 484p. (Princípios do tratamento biológico de águas residuárias; 6).

SANEPAR - Companhia de Saneamento do Paraná. Manual técnico para utilização agrícola do lodo de esgoto no Paraná. Curitiba: SANEPAR, 1997. 96p. 